\title{
Microdosing as a response to the meaning crisis: a qualitative analysis
}

\author{
Rotem Petranker ${ }^{*}$, Juensung Kim², Thomas Anderson3
}

Background: The use of psychedelic substances like LSD and magic mushrooms in research and to treat mental health conditions has been increasing in the last decade. In particular, the practice of microdosing - using sub-hallucinogenic amounts of psychedelics - has been increasing

(Anderson et al., 2019), but large-scale systemic qualitative analyses are still scant.

Aims: This study attempted to recognize emergent themes in qualitative reports regarding the experience of microdosing so as to enrich the theoretical landscape in psychedelics research and propose future research directions for both basic and clinical research.

Methods: This study used qualitative analysis to analyze free-text responses from individuals who participated in an online survey disseminated on various social media platforms. Participants had reported microdosing at least once in the past year.

Results: Data from 118 informative responses suggested four main emergent themes: reasons for microdosing, the practice of microdosing, outcomes linked to microdosing, and meta-commentary about microdosing. Participants mostly reported microdosing for clinical reasons and to improve productivity, and mentioned that the practice is often challenging due to unknown optimal dosing regimen. The outcomes of microdosing varied widely between strong endorsement of the practice and disappointment at the lack of effect. Meta-commentary included warning against overexcitement with the practice. We couch our findings in meaning-making theory and propose that, even at low doses, psychedelic substances can provide a sense of meaning currently lacking in Western culture.

Conclusion: Our results suggest that there many of the reported benefits occur regardless of motivation to microdose and are likely due to the enhanced psychological flexibility and sense of connectedness made possible due to the use of psychedelics. Double-blind, placebo controlled experiments are required in order to substantiate these reports.

Mental illness is a growing global concern: depression and anxiety are currently the 5th leading cause of disabilityadjusted life years lost in North America (Murray et al., 2012). Rigid patterns of negative thoughts and deficits in creative, flexible thinking have been shown in stressrelated illnesses like depression and anxiety (Gabrys, Tabri, Anisman \& Matheson, 2018; Morrison et al., 2016; Cusi et al. 2011; Donges et al. 2005). Psilocybin and other psychedelics have been linked to reducing anxiety and depression (Tupper, Wood \& Johnson, 2015) and have also been shown to promote creative thinking and creative problem-solving (Anderson et al., 2019; Prochazkova et al., 2018; Sessa 2008; Harman et al.,1966). These effects are thought to be mediated by two mechanisms: first, through the physical and chemical changes taking place in the brain via serotonin receptors (Krebs-Thomson et al, 1998; Vollenweider et al., 1998; Krebs-Thomson and Geyer, 1996), and second, through the psychological changes brought about by profound spiritual insights gained from psychedelic-induced peak mystical experiences (Griffiths et al., 2011).

At higher doses, psychedelic substances affect perception and introduce unusual sensory experiences, potentially occasioning mystical experiences, existential insights, and feelings of connectedness within oneself, with others, and with nature (Schmid et al., 2015; Studerus,
1 Department of Psychology, York University, Toronto, ON, Canada.

2 Department of Applied Psychology and Human Development, University of Toronto, Toronto, ON, Canada.

3 Department of Psychology, University of Toronto, Toronto, ON, Canada.

*Corresponding Author e: rotem@boredomlab.org 
Kometer, Hasler, \& Vollenweider 2011; Griffiths, Richards, Johnson et al., 2008; Griffiths et al., 2011). Promoting feelings of connection may be particularly valuable in contemporary secular society wherein people often suffer from a feelings of isolation and meaninglessness (Anderson, 2010; Jenkins, 2018; Sliwa, 2017; Vervaeke \& Miscevic, 2017).

Family and religion are two factors that can be protective of mental health concerns, whether by providing interpersonal support networks and community structures for social interaction or by offering frameworks and strategies for meaning-making and purpose (Park, 2017; Pernice-Duca, 2010). While other factors also exist, especially for younger generations (Lukianoff \& Haidt, 2015,2018 ), the declining presence of protective factors, like strong social communities, likely contributes to rising mental health concerns (Hartogsohn, 2018) and what has been labelled a "loneliness epidemic" (Snell, 2017).

Isolation, loneliness, and disconnection from social communities are most deeply entrenched in treatmentresistant mental health disorders (Hawkley \& Capitanio, 2015); remarkably, mounting evidence suggests that high doses of psilocybin and other psychedelic substances can be part of effective treatment pathways for these disorders (Carhart-Harris et al., 2017; Osorio, Sanches, \& Macedo et al., 2015). Unlike conventional therapies, psychedelics can work directly to increase inter-connectivity in networks throughout the brain, physically promoting new connections that, in combination with therapy, appear to help overcome unhealthy, negative thought patterns by fostering new pathways and insights (Carhart-Harris et al., 2019; Carhart-Harris et al., 2017). Psychedelics have also been associated with long-term positive psychological wellbeing in the form of increased openness, cognitive flexibility, and creative thinking (MacLean et al., 2011; Prochazkova et al., 2018).

While potentially useful, mystical experiences induced by high dose psychedelics drastically alter the acute perception of reality, seriously impeding normal functions during the experience. Activities of daily life — such as driving, working, or caregiving - are impossible while under the acute effects of a high dose of psychedelics. In contrast, microdosing psychedelics - the practice of taking small, sub-hallucinogenic doses of substances like psilocybin-containing mushrooms or lysergic acid diethylamide (LSD) — does not appear to impair normal functioning, yet users still report mental health benefits (Anderson et al., 2019). Recent work suggests that, despite unaltered perception and lack of mystical experiences, microdosing psilocybin is associated with improved mood and increased creativity (Anderson, Petranker, Rosenbaum, et al., 2019a; Polito \& Stevenson, 2019; Prochazkova et al., 2018).

Perhaps due to the minimal apparent drawbacks of microdosing (Anderson, Petranker, Christopher, et al., 2019), this practice has been growing rapidly, as evidenced by publications in the popular press and the growth of online communities dedicated to microdosing. For example, membership in the Reddit microdosing community has grown by a factor of 20 over the past 4 years, from $5 \mathrm{k}$ subscribers in 2016 to over $100 \mathrm{k}$ subscribers as of early 2020 (Reddit.com/r/microdosing). Microdosing psychedelics represents a potential complementary approach for addressing several mental health conditions, ostensibly without impairing function. However, microdosing lacks a phenomenological theoretical framework in which to situate any salutary effects. While it is possible that the impact of microdosing psychedelics is merely chemical, we seek also understand what needs this practice serves. To this end, we used data from an existing online survey to analyze participants' free-text reported experience with microdosing

\section{Methods}

Participants were recruited mostly through posts on the online forum "reddit" (Reddit Inc, San Francisco, CA, USA). Links were posted under the username /u/oredna on the following subreddits: Microdosing, Nootropics, Psychonaut, RationalPsychonaut, Tryptonaut, Drugs, LSD, shrooms, DMT, researchchemicals, and SampleSize. Participation was voluntary and participants were not paid for their participation. The survey was in English and available only online. Not all participants who started the survey completed it: while 1390 respondents began the survey, 475 exited before responding, 3 requested that their responses be removed, and 3 responses were removed for intentionally factitious responding ("trolling"). At the end of the survey, open-ended commentary regarding microdosing was solicited from participants, using the prompt, "If there are any other comments you would like to leave about microdosing please share them here!" Results from the rest of the dataset collected in this survey have been published (T. Anderson, Petranker, Christopher, et al., 2019, 2019; Rosenbaum et al., 2020).

Data were exported to a spreadsheet and two relevant columns were extracted: Participant ID and the column containing responses to the above prompt. Of the 909 total participants in the study, 170 participants provided commentary in the space provided. Of these, 28 participants were excluded from the present analysis for providing commentary consisting only of a call for the legalisation of psychedelics, volunteering for participation in further research, or both. As we are here interested in participants' experience of microdosing itself, these comments were considered unsuitable to the present analysis and therefore excluded, leaving 142 participants. These remaining reports were compiled into a single document and imported into QSR's NVivo 12 Plus (QSR International Pty Ltd.). Of these 142 participants that provided comments, a further 24 participants were excluded from analysis after the completion of coding: 16 for identifying themselves as nonmicrodosers in the quantitative portion of the survey, 4 for revealing through their comments that their microdosing experience was minimal, 2 for providing comments on answers to the survey in an inappropriate box, and 2 for providing other forms of irrelevant commentary. The final sample included 118 participants (Table 1).

Coding was conducted by the second author, a researcher in lifespan developmental psychology trained in qualitative analysis and previously unfamiliar with research literature on psychedelics to avoid potential bias. Coding proceeded following the methods of interpretive 
phenomenological analysis (IPA; Smith, Flowers, \& Larkin, 2009). Due to the unconstrained method of data collection giving participants freedom to report whatever they felt was most important to say, as well as the interest of the present paper in capturing the lived experience of microdosing, IPA was considered the most appropriate method to use to best analyse participants' reports. Following Smith, Flowers, and Larkins (2009) guidelines for IPA with larger sample sizes, the analysis focused on understanding commonalities among participant's experience of their microdosing.

Analysis began with a thorough reading of participants reports, with coding categories emerging through a process of abstraction with NVivo used to manually sort opencoded units, identified on the second readthrough, into groups of similar topics or themes. Progressively higherorder coding categories emerged from these lower-order categories as thematic groups were themselves filed into groups. These groups were ordered into categories pertaining to the nature of the commentary, which serve as the primary themes uncovered (see Table 2).

Analysis of the 118 included participants produced 123 individual themes, which were then grouped into primary themes and a number of subheadings within each (see Table 2). Participants who provided relevant commentary generally reported on one or more of four aspects of their experience of microdosing: their reasons and motivations for microdosing, the technical details of their microdosing practice, reported outcomes from microdosing, and meta-commentary on microdosing in general. These have been organised for presentation below in the above order, rather than by frequency, to better approximate a narrative format conducive to understanding the experience of microdosing psychedelics.

\section{Reasons for Microdosing}

The most common reasons given for taking up microdosing were clinical in nature, especially for mood disorders. Addiction treatment, suicidality, trauma, chronic pain, and intrusive thoughts were all also reported as reasons for microdosing. Four participants explicitly noted that they microdosed psychedelics as a substitute for conventional medicine.

"Charlie": "I've been depressed for my entire adolescence, SSRI's helped a lot with my anxiety but they didn't do anything for my depression. the first time I microdosed I felt like I stopped being depressed for the first time in my life, before that I didn't even knew that I was that depressed. that feeling lasted until I got depressed almost a year after. I'm on a different antidepressant at the moment and it seems to help a lot but if I could get my hands on at least one tab of LSD I think id be much less depressed by now, but LSD is really hard to come by here.

The next most commonly reported reasons for microdosing were for productivity and to facilitate novel or creative thought. Schoolwork was explicitly noted by two participants. Participants also reported using microdosing to effect more permanent changes in their perspectives than single bursts of novelty, seeking to improve their ability to take other perspectives, change their personalities, or grow as people. Others used microdosing to improve their social aptitude or gain a deeper "insight into life". Interestingly, only one participant explicitly stated that they microdosed for fun. Though hedonistic themes do arise in participants' comments, they are mentioned largely as a possible side effect or result of difficulties calculating proper doses.

"Skylar": "I have never micro dosed on a schedule (i.e every four days) but do it either as a study/work enhancer if I know I have a lot to do in a day (which is often as a student) such as before exams or when I know I am going to have a very enjoyable day doing things I already love like biking and hiking to add to the experience. Tomorrow is a combination where I have a lot of work in the morning, and a fun bike ride planned in the afternoon. I will be microdosing. I have tried prescription study drugs, and although there is more energy with those like Adderall, they make me feel jumpy and my mind feels fuzzy and out of focus. Microdosing is far better for me"

This pattern of microdosing to improve one's experience of life, either due to symptom amelioration or to achieve a fuller, more varied perspective, will reemerge when discussing participants' reported outcomes of microdosing.

\section{Practice of Microdosing}

The most frequently provided commentary regarding participants' microdosing practice involved the doses of various substances and the timing of their administration. The former was particularly emphasised as participants repeatedly described the difficulty of calculating the correct dose for their height, weight, and tolerance that would provide positive effects without resulting in hallucinogenic effects. In some cases, this would prove so difficult that participants report giving up microdosing. One participant reported having microdosed for four months, combined with meditation and therapy, and not finding any effects. This may be due to miscalculation of dose, resulting in negligible or no effects at all.

"Alex": "I gave an estimate for how frequently I dose (once per week), but it is not so regular. Sometimes I go for months without, and sometimes I microdose twice in a week. But I usually go through periods where I microdose more frequently, and during those periods I would say once per week is about right."

"Blair": "I am sad to report that after 4 months of following Fadiman's protocol, and in addition to daily meditation and weekly therapy, I have not noticed any benefits, neither has my wife of 26 years." 


\section{Preprint}

\section{Table 1}

Demographics of the included 118 participants.

\begin{tabular}{|c|c|c|}
\hline Age (years) & Mean (SD) & $27.58(9.17)$ \\
\hline & Median & 25 \\
\hline & Range & $17-65$ \\
\hline & Prefer not to answer / NA & 3 \\
\hline \multicolumn{3}{|l|}{ Gender } \\
\hline & Male & 99 \\
\hline & Female & 17 \\
\hline & Neither/Non-binary & 1 \\
\hline & Prefer not to answer / NA & 1 \\
\hline \multicolumn{3}{|c|}{ Sexual orientation } \\
\hline & Heterosexual & 88 \\
\hline & Homosexual & 3 \\
\hline & Bisexual / Multisexual & 24 \\
\hline & asexual & 1 \\
\hline & Prefer not to answer / NA & 2 \\
\hline \multicolumn{3}{|c|}{ Ethnic heritage } \\
\hline & White/European & 91 \\
\hline & Black & 0 \\
\hline & East Asian & 1 \\
\hline & Middle Eastern & 1 \\
\hline & Hispanic or Latino & 5 \\
\hline & Mixed & 9 \\
\hline & Other & 6 \\
\hline & Prefer not to answer / NA & 5 \\
\hline \multicolumn{3}{|c|}{ Religious affiliation } \\
\hline & Non-religious, atheist, or agnostic & 64 \\
\hline & Judaism & 4 \\
\hline & Christianity & 5 \\
\hline & Islam & 0 \\
\hline & Hinduism & 1 \\
\hline & Buddhism & 5 \\
\hline & Other religious affiliation & 4 \\
\hline & Spiritual but non-religious & 32 \\
\hline & Prefer not to answer / NA & 3 \\
\hline \multicolumn{3}{|l|}{ Social class } \\
\hline & Upper-middle class & 16 \\
\hline & Middle-class & 44 \\
\hline & Lower-middle class & 24 \\
\hline & Skilled working class & 10 \\
\hline & Working class & 11 \\
\hline & Non-working class (casual workers, pensioners, or dependants) & 9 \\
\hline & Prefer not to answer / NA & 4 \\
\hline \multicolumn{3}{|l|}{ Education } \\
\hline & Doctoral or equivalent & 3 \\
\hline & Master or equivalent & 17 \\
\hline & Bachelor or equivalent & 37 \\
\hline & Short-cycle tertiary (Associate degree, Advanced Diploma) & 12 \\
\hline & $\begin{array}{l}\text { Post-secondary non-tertiary (Certificates, Diplomas, or partial completion of a } \\
\text { degree) }\end{array}$ & 23 \\
\hline & Upper secondary or less & 27 \\
\hline & Prefer not to answer / NA & 4 \\
\hline
\end{tabular}




\begin{tabular}{|l|c|l|l|c|c|}
\hline Theme & Participants & $\begin{array}{l}\text { Total } \\
\text { References }\end{array}$ & Sub-theme & Participants & $\begin{array}{l}\text { Total } \\
\text { References }\end{array}$ \\
\hline $\begin{array}{l}\text { Reasons for } \\
\text { Microdosing }\end{array}$ & 28 & 46 & Clinical purposes & 21 & 31 \\
\hline & & & Productivity purposes & 3 & 3 \\
\hline $\begin{array}{l}\text { Practice of } \\
\text { Microdosing }\end{array}$ & 47 & 61 & Information about dose and timing & 23 & 23 \\
\hline & & & Microdosing Schedule & 16 & 17 \\
\hline & & & Use in Combination & 10 & 14 \\
\hline $\begin{array}{l}\text { Outcomes of } \\
\text { microdosing }\end{array}$ & 62 & 125 & Existential Effects & 35 & 45 \\
\hline & & & Clinical Effects & 26 & 33 \\
\hline & & & Cognitive Effects & 15 & 19 \\
\hline & & & Performance Effects & 10 & 13 \\
\hline $\begin{array}{l}\text { Meta-commentary on } \\
\text { microdosing }\end{array}$ & 27 & 32 & Interpersonal Effects & 10 & 10 \\
\hline
\end{tabular}

Table 2

Number of

participants and total references per theme, with major subthemes
The use of microdosing in combination with other practices or substances was also a common theme when reporting on participants' practices, with 10 participants reporting microdosing in combination with other practices. Microdosing was often reported as being used in combination with meditation, exercise, therapy, dietary changes, or a combination of the above as part of a selfimprovement regimen. In contrast, microdosing was sometimes combined with other performance-enhancing substances, such as caffeine, ephedrine, marijuana, or Noopept (N-phenylacetyl-L-prolylglycine ethyl ester, a synthetic nootropic molecule), especially when aims were specific to clinical or productivity-related domains rather than major life changes. One participant instead reported reduced effects when microdosing under the influence of marijuana.

"Casey": "I had tremendous success in recently treating decades of aftermath of long term abuse, trauma and loss with full dose psychedelic medicines. I am microdosing now for maintenance in conjunction with talk therapy and it has been nothing short of miraculous for me."

Concerning timing, the majority of participants who discussed the scheduling of their microdoses reported taking them irregularly, usually as they felt they needed a boost to mood or performance. Participants who reported a regular dosing schedule ranged from approximately every other day to weekly; in particular, participants reported not microdosing every day. Avoiding daily microdosing could be a preventative measure to avoid building tolerance to the substance. As one participant noted, tolerance to microdosing can be built rather quickly, which is in agreement with the literature (Nichols, 2016). Additionally, any increase in tolerance would exacerbate reported difficulties in calculating useful doses.

Finally, four participants observed that the results of a microdose can be strongly tied to one's mindset, with one additional participant noting the importance of a supportive environment for a successful practice. This finding is in agreement with the emphasis in lay and scientific contexts on "set and setting" (Hartogsohn, 2016). These participants reported that microdosing opened and amplified their experience of the world, but any insights drawn had to be under the person's own power:

"Finley": "I feel that microdosing has a lot to do with how you feel inside at the moment and/or what you wish to achieve at the moment. I feel that psychedelics amplify, in a way, our current feelings/ needs/wants. SO if people are feeling depressed or anxious, I believe that they shouldn't do it if they don't know what they are getting into, because for some weak hearted, it might trigger something that will be beyond their control. Although overall I am definitely convinced of the therapeutic power of psychedelics, psilocybin mushrooms to be exact, but I also know that some people cannot handle them."

\section{Outcomes of Microdosing}

Consistent with reasons for microdosing, participants frequently reported clinical benefits, particularly regarding mood disorders, stress, and addiction.

"Devon": "The best period of my life seemed while I microdosed semi-frequently. my psychiatric problems (depression, anxiety and drug mis-use) were diminished. I stayed sober, active, happy and created a lot."

Interestingly, despite optimistic reports of microdosing having no negative side-effects, some participants reported that microdosing caused or exacerbated clinical symptoms, particularly anxiety and depersonalisation. Two participants reported "come-down effects", one participant decreased motivation, and two increases in stress. It should be 
acknowledged that such reports were proportionally in the minority and seem to resonate with the above warnings regarding the importance of one's mindset when microdosing

"Jamie": "I am a grad student who works in a lab, and have md'd on days where I was doing rote tasks. I found $\mathrm{md}$ to make these tasks a little more enjoyable, likely due to my overall mood increase. One day while I was $\mathrm{md}$, something unexpected happened that required more critical thinking and dexterity than a typical lab day. I found that things were amplified now in a more negative way - I was more stressed about the event, and more anxious about making mistakes."

"Ashleigh": "Psychedelics tended to make me depersonalized long after I took them. Even in microdoses."

Benefits to performance, or to cognitive capacities in general, were also reported, again in keeping with some of the stated reasons participants practiced microdosing. A clearer mind, improved focus, and greater creativity were some of the more common such benefits, although the above-quoted participant noted impairment to critical thinking skills when placed in an unexpected and stressful situation (See quote above, "Jamie"). More specific enhancements included reading comprehension, spatial awareness, and abstract comprehension, along with one participant noting significantly improved athletic prowess while microdosing.

"Lee": "Microdosing LSD worked incredibly well playing rugby. Lightning fast reflexes, on-point ballflight-path calculations and strategic insight like Lord Admiral Nelson was whispering directions into my ear."

Interestingly, two participants noted the effects of microdosing on the vividness of dreams, with one participant reporting taking it as an aid to lucid dreaming practice. Improvements in motivation and enjoyment of work were also mentioned. Participants also reported improvements in interpersonal interaction, finding social functioning more fluid, with a deeper sense of interpersonal connection.

"Mackenzie": "Microdosing is a pretty good way to get out of your shell and socialize with people, in my opinion. I'm not usually the one to drive conversation out of people, but on LSD it's almost too easy not to."

While many reports were in keeping with reported reasons for microdosing - such as symptom-reduction, productivity, or general enhancement - the largest share of reported experiences (45) concerned the existential effects of microdosing. Participants reported a generally improved quality of life as a result of microdosing, greater selfacceptance, and a deeper appreciation of life. Participants reported an increased sense of awareness of themselves and their relationship to both others and the world, while finding it easier to live in the moment and let things go. Additionally, reports of a feeling of calm, connection, and self-acceptance were common when describing the experience of microdosing.

"Morgan": "I believe the greatest thing I learnt from microdosing was to stop trying to solve problems I perceived about myself and just accept them for what they were. Accept myself for who I am, relax, stop overthinking everything because at the end of the day none of this matters so you may as well enjoy yourself however you see fit."

\section{Meta-commentary on Microdosing}

The larger share of meta-comments about microdosing were reserved or skeptical about the benefits with 11 participants providing negative commentary. They reported that the effects of microdosing psychedelics were minimal, lacking the effects found at larger doses, or that microdosing was not for everyone. One participant observed caffeine had a greater effect for them. Another warned that microdosers risk feeling too good, i.e. indulging in hedonism rather than increasing productivity.

"Kadin": "Microdosing can provide some mild effects, both positive and negative. After experimenting with it a little bit, I'm not very impressed. Caffeine is much more useful for providing the benefits often attributed to microdosing psychedelics."

"Harley": "One of the 'dangers' of microdosing is that you feel so good that you think 'I have to use this, I want to do more fun things than study.' Another is that your mind gets too far open and you start to become soo sad when thinking about the state of the world and all the suffering everywhere."

Contrasting these concerns were nearly as many expressions of optimism regarding microdosing, with ten participants providing positive comments. These participants asserted that benefits could be long-lasting and that negative side-effects were few, making them possibly safer than higher doses of psychedelics despite the acknowledged loss of some of the effects of a larger dose. Two participants expressed a hope that education regarding effective microdosing would one day become more widespread.

"Cameron": "Microdosing is a powerful tool to gain insight on your life as well as re-program aspects of you personality in a way that causes no side effects other than increased tolerance to the drug of choice. I highly recommend everyone try it."

"Taylor": "Microdosing is definitely a very beneficial thing that nearly anyone in a position to 
take advantage of its creative potential, whether for work or personal expression. It should be respected and not over-used or leaned upon but used as a tool of productivity and stretcher of personal potential. I believe some day we'll look at microdosing in a similar way that we do today with coffee. Enjoyable and a productivity enhancer."

Where participants discussed using more than a single type of psychedelic compound, particularly LSD or psilocybin-containing mushrooms, they would often note differing effects. In particular, LSD was observed to be more stimulating, leading to increased energy, focus, and creativity, while psilocybin was reported to facilitate greater openness and calm. These differences were occasionally experienced negatively, particularly in the case of one participant who reported that the only effect psilocybin had on them was drowsiness. Finally, a few participants made comparisons between the effects of microdosing psychedelics and other commonly used stimulants: coffee (caffeine) or Adderall.

\section{Discussion}

We have extrapolated across themes present in participant reports to assemble an overarching descriptive narrative of common reasons to start microdosing. Our findings suggest two complimentary narratives: a clinical narrative, which frames microdosing as a form of selfmedication for pain or mental health complaints, and a flourishing narrative, which frames microdosing as part of a regimen for self-improvement. These two narratives presage two different framings of acute reported benefits (Figure 1).

Microdosing was reported to be a response to various mental health issues or to enhance creativity and productivity. Specifically, reasons for microdosing were most often symptomatically motivated - e.g. for depression, anxiety, low mood, or pain - or as part of an overall program wherein participants were trying to improve their lives beyond healthy levels. Curiously, outcomes of microdosing were often broader in scope: providing greater perspective, increased sense of connection to others, or a sense of calm and self-acceptance. Microdosers started for one reason, but they report getting broader benefits than initially intended.

Acute reported benefits are in accord with the existing literature with the coexistence of clinical and flourishing narratives acting as a common theme during the first wave of psychedelic research in the 1950s and 60s (Grinspoon \& Bakalar, 1979). Our sample also hints that both symptom reduction and self-improvement may share a cumulative distal outcome of enhanced sense of connectedness with others. This unanticipated outcome reflects a shared benefit reported across both the clinical and flourishing narratives.

\section{Two Narratives}

Considering first the clinical narrative, we note that microdosers were motivated to self-treat their mental health concerns including anxiety, depression, suicidality, or social anxiety. Participants largely reported being successful in their self-treatment goals with many reporting reduced depression and anxiety, though not universally as some reported increased anxiety, especially related to dose inaccuracy. Psychedelics at larger doses show promise as effective treatment options for mood disorders (e.g. depression, (Carhart-Harris et al., 2017), obsessive compulsive disorder (Moreno et al., 2006), anxiety disorders (dos Santos, Bouso, Alcázar-Córcoles \& Hallak, 2018), and substance use disorders (Bogenschutz et al. 2015; Johnson et al. 2014) when taken in a controlled environment. Our findings suggest that beneficial treatment options may also exist even at the level of a microdose, while decreasing the risk of the "bad trip" (Carbonaro et al., 2016) and significantly lowering the costs related to long, in-patient sessions with up to two therapists at once, which would make microdosing much more financially feasible than higher-dose psychedelic psychotherapy. Indeed, taking the participants at face value, the most common negative outcome with psychedelic microdosing was that nothing happened. Most negative reactions were paired with dosing inaccuracy, either too high or too low, which could be alleviated by proper experimental control and/or legal regulation. Curiously, self-medication research reports that most proponents of self-medication for mood and anxiety disorders are young white men (Turner et al., 2018), a demographic which also forms the majority of respondents to the present study and that may be underserved by the present clinical treatment model for one reason or another.

Regarding the flourishing narrative, microdosers aimed at self-improvement, often with regards to creative output or productivity, and reported being successful in their attempts. Despite empirical limitations, a growing theoretical literature considers whether larger doses of psychedelics may improve well-being in non-clinical populations. For example, one theory paper suggests that psychedelics could enhance well-being following a similar mechanism to that of profound meditative experiences (Millière et al., 2018). Another line of inquiry has proposed that psychedelics may help shift subjective meaning-making foci (Hartogsohn, 2018). Finally, the REBUS model suggests that psychedelic use flattens the phenomenological landscape through which consciousness travels, enabling thinking in novel patterns (Carhart-Harris \& Friston, 2019). All three theories hypothesize that using psychedelics would allow one to think more flexibly and creatively, which agrees with the scant empirical evidence available (T. Anderson, Petranker, Rosenbaum, et al., 2019b; Prochazkova et al., 2018b). In our sample, reported benefits of microdosing for flourishing purposes focused mostly on creativity and enhanced focused attention. These data do not appear to discriminate between the aforementioned theoretical accounts, which are not mutually exclusive. Future research on microdosing should not limit itself to clinical measures alone; measures of flourishing and "euthymia" (Fava \& Bech, 2016) should be included to capture more rarefied benefits to human wellbeing.

\section{Shared Benefits}

Irrespective of whether their narrative was focused on clinical or flourishing benefits, participants reported microdosing was related to the ability to take their problems in perspective, decreased identification with thoughts and feelings, and paying attention to disparities between one's 
values and behavior. These reported benefits correspond to some of the fundamental principles of Acceptance and Commitment Therapy (ACT), namely self-ascontext, defusion, and value-oriented action, all a part of psychological flexibility (Hayes et al., 2011). ACT theory predicts that improvement on these dimensions should improve well-being trans-diagnostically, and recent metaanalyses suggest that this may indeed be the case (A-Tjak et al., 2015; Ruiz, 2012). ACT has also provided foundational inspiration to the most commonly used therapy protocol for lab-based large-dose use of psychedelics, suggesting that psychedelics may enhance psychological flexibility, which in turn improves well-being (Watts \& Luoma, 2020). The theory explaining the usefulness of ACT for large-dose use of psychedelics may also be relevant for microdosing, and specifically for the shared benefits reported regardless of clinical or flourishing narratives.

In our sample, participants reported increased psychological flexibility after microdosing without ACT or any other kind of therapy. Examples of benefits reported include a broader perspective wherein little problems are less troublesome; less overthinking; and an awareness of choices and their alignment with personal values. Similar benefits have been described for large-dose use of psychedelics (Carhart-Harris et al., 2018) and microdosing (Lea et al., 2019), but a fleshed out theoretical link remains missing. For example, the REBUS model suggests that use of psychedelics enhances psychological flexibility, but draws exclusively on studies that included large-dose use of psychedelics primarily in the context of therapy. In our sample, however, psychological flexibility was reported to be enhanced by microdoses without therapy. This finding leaves two questions open: first, is therapy necessary for psychological flexibility brought about by psychedelics? Second, could microdosing over time be as effective as single large-doses in increasing psychological flexibility? Psychedelic therapy could be far less onerous to implement if hallucinogenic doses and/or therapy are not necessary to increase psychological flexibility, especially if psychological flexibility proves to be a primary moderator of improved well-being as predicted by the ACT model. Future research could examine whether a purely

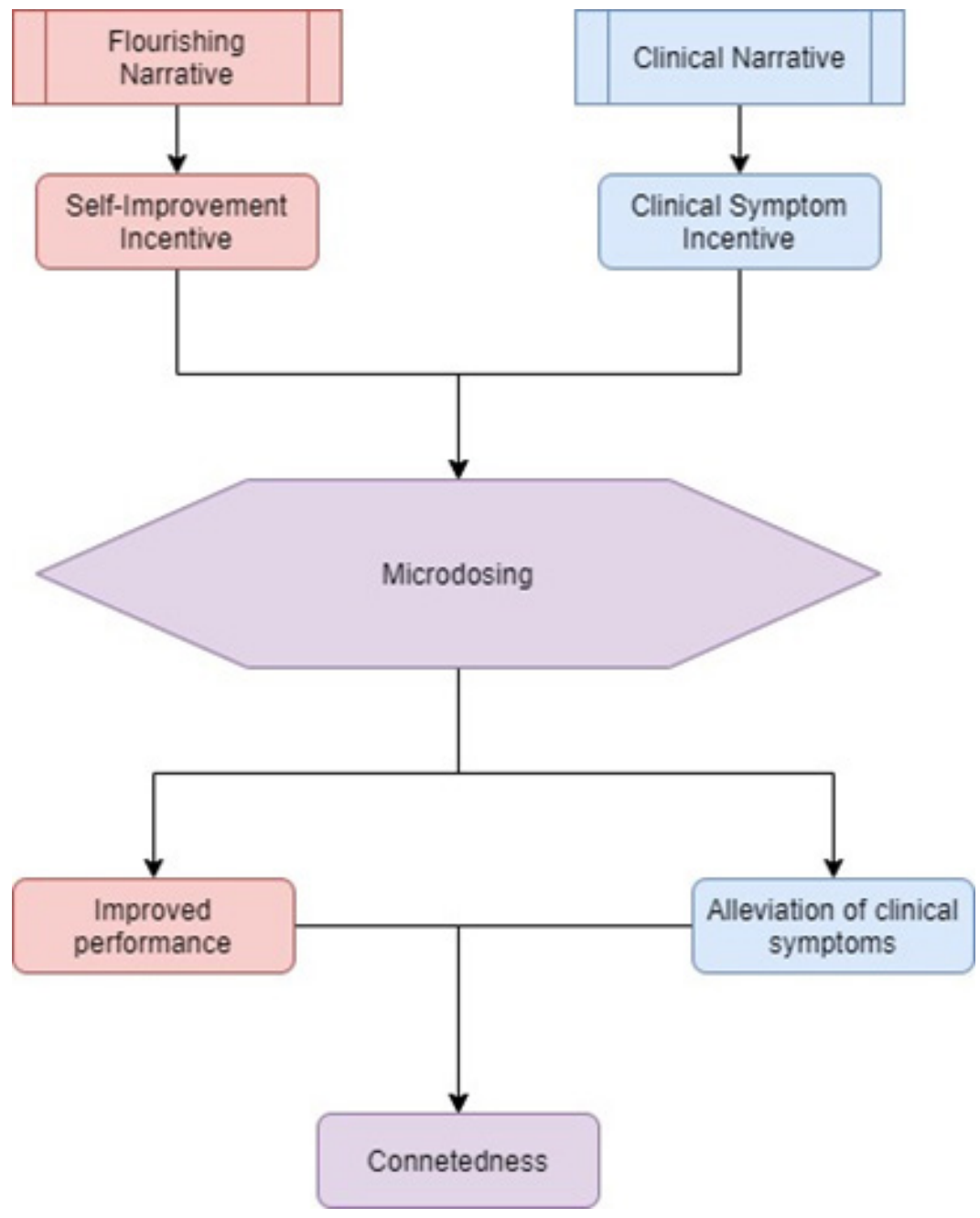

\section{Figure 1}

Narrative pathways from initial motivations through microdosing to acute and cumulative outcomes. 
pharmacological intervention also improves well-being in clinical and nonclinical populations. Another potential research avenue would involve strict ACT therapy, which focuses on increasing psychological flexibility, rather than an ACT-inspired perspective. Future research could examine these hypotheses about the shared benefits experienced by microdosers.

\section{Connectedness and Sense of Meaning}

We speculate that the aforementioned shared benefits could be indications of an increased sense of meaning, purpose, or goal-directedness in life (Carhart-Harris et al., 2018; Costin \& Vignoles, 2019). While mystical experiences are hypothesised to be a primary mediator of the increased sense of meaning and purpose following larger psychedelic doses (Hendricks, 2018), it is of note that similar gains appear to be possible through microdosing, which does not give rise to the same phenomenological effects (Haijen, 2018; Yaden et al, 2017).

Microdosing may not have these effects for everyone. Some individual differences could be useful in predicting who would be more likely to benefit from microdosing. One promising individual difference is trait absorption, which positively predicts a search for meaning in life (Steger et al., 2008) and predicts both likelihood of mystical experiences and well-being improvement following ingestion of higher doses of psychedelic compounds (Haijen et al., 2018). Mystical experiences themselves are also correlated to experiencing meaning in life (Kim \& Vervaeke, 2019). We recommend measuring the effect of trait absorption in future research on psychedelic microdosing.

According to the World Health Organization, prevalence of depression and anxiety disorders globally increased by $18.6 \%$ and $14.9 \%$ between 2005 and 2015 (WHO, 2017). While one possible explanation for this is technology and urbanisation-driven changes in global lifestyle leading to physiological symptoms that overlap with stress, such as cardiovascular disease and fatigue (Baxter et al., 2015), there is an increase in major depressive episodes, suicide, and suicide attempts since 2014, in the generation born during the 1990s as well as those of the "Boomer" generation born in the 1950s (Twenge et al., 2019). The last century presented a marked increase in the proportion of people living alone and a corresponding increase in the prevalence of feelings of loneliness, isolation, and disconnection (Snell, 2017). More recently, society has seen a decrease in the amount of in-person social interaction and concomitant increase in the amount of social media use, particularly in the generation born in the 1990s. Feelings of loneliness and isolation are strongest in those high in social media use and low in in-person interaction (Twenge, Spitzberg, \& Campbell, 2019).

Accompanying the increase in single-person households and decline of in-person interaction has been a decline in membership to religious communities, (Franck \& Iannaccone, 2014; Twenge et al., 2016), which have historically provided environments supporting in-person interactions and socially sanctioned pathways to meaning (Park, Edmondson, \& Hale, 2013). Eberstadt (2013) has argued that the decline of religion and the rise in singleperson households are intimately connected, but reverses the more common narrative of a decline in religion leading to a decline in traditional family structure. Rather, Eberstadt argues that the changing social landscape of the last several centuries has resulted in people increasingly living apart from family, and that this change in family structure was instrumental in the decline in religious participation. Without the supporting structure of family tradition to reinforce it, religious practice falls away, and with the loss of both family- and religion-based social connections comes the increase in feelings of loneliness, isolation, and disconnection (Bonn \& Taferodi, 2013).

Considering the reports of an increased sense of connectedness to themselves and to others following microdosing, it seems that some people find microdosing may alleviate some of the apparent societal loss of connection and meaning. As argued by Hartogsohn (2018), psychedelics have historically been a powerful tool for altering the perception of meaning. Hartogsohn argues that the phenomenology of psychedelics stands in direct contrast to current first-line antidepressants in that they amplify, rather than dampen, the intensity of experience. This experience-amplifying function appears to be at the core of their role in spiritual, therapeutic, and creative work: the increased vividness that accompanies psychedelic experiences allows for positive re-evaluation of existing social connections and realizing new meaning in old ideas or phenomena. Psychedelics, even at low doses, may help promote new ways of thinking about and looking at the world and one's place within it.

\section{Conclusions}

In our sample, microdosing psychedelics was often driven by a will to treat clinical issues or to enhance flourishing. While most participants approached microdosing with a local intention, many reported both local and global benefits. Judging from this sample, as well as the growing literature on microdosing, it is plausible that smaller doses of psychedelics over time, situated within a context of the active pursuit of personal development, may have similar effects to larger dose, all with decreased risk of adverse reactions. Future research should investigate the potential of lower doses of psychedelics, assessing the potential challenges associated with using these substances in small doses, and explore ways to optimize the potential benefits reaped from using psychedelic substances.

\section{References}

Anderson, G. O. (2010, September). Loneliness Among Older Adults: A National Survey of Adults 45+. AARP. https://doi.org/10.26419/ res.00064.001

Anderson, T., Petranker, R., Rosenbaum, D., Weissman, C. R., DinhWilliams, L.-A., Hui, K., Hapke, E., \& Farb, N. A. S. (2019a). Microdosing psychedelics: Personality, mental health, and creativity differences in microdosers. Psychopharmacology, 236(2), 731-740. https://doi. org/10.1007/s00213-018-5106-2

Anderson, T., Petranker, R., Rosenbaum, D., Weissman, C. R., DinhWilliams, L.-A., Hui, K., Hapke, E., \& Farb, N. A. S. (2019b). Microdosing psychedelics: Personality, mental health, and creativity differences in microdosers. Psychopharmacology, 236(2), 731-740. https://doi. org/10.1007/s00213-018-5106-2

A-Tjak, J. G. L., Davis, M. L., Morina, N., Powers, M. B., Smits, J. A. J., \& Emmelkamp, P. M. G. (2015). A Meta-Analysis of the Efficacy of Acceptance and Commitment Therapy for Clinically Relevant Mental and Physical Health Problems. Psychotherapy and Psychosomatics, 84(1), 30-36. https://doi.org/10.1159/000365764

Carhart-Harris, R. L., Erritzoe, D., Haijen, E., Kaelen, M., \& Watts, R. 
(2018). Psychedelics and connectedness. Psychopharmacology, 235(2), 547-550. https://doi.org/10.1007/s00213-017-4701-y

Carhart-Harris, R. L. \& Friston, K. J. (2019). REBUS and the Anarchic Brain: Toward a Unified Model of the Brain Action of Psychedelics. Pharmacological Reviews, 71(3), 316-344. https://doi.org/10.1124/ pr.118.017160

Fava, G. A., \& Bech, P. (2016). The Concept of Euthymia Psychotherapy and Psychosomatics, 85(1), 1-5. https://doi. org/10.1159/000441244

Hartogsohn, I. (2016). Set and setting, psychedelics and the placebo response: An extra-pharmacological perspective on psychopharmacology. Journal of Psychopharmacology, 30(12), 1259-1267. https://doi. org/10.1177/0269881116677852

Hartogsohn, I. (2018). The Meaning-Enhancing Properties of Psychedelics and Their Mediator Role in Psychedelic Therapy, Spirituality, and Creativity. Frontiers in Neuroscience, 12. https://doi.org/10.3389/ fnins.2018.00129

Hawkley, L. C., \& Capitanio, J. P. (2015). Perceived social isolation, evolutionary fitness and health outcomes: A lifespan approach. Philosophical Transactions of the Royal Society B: Biological Sciences, 370(1669), 20140114. https://doi.org/10.1098/rstb.2014.0114

Hayes, S. C., Strosahl, K. D., \& Wilson, K. G. (2011). Acceptance and Commitment Therapy, Second Edition: The Process and Practice of Mindful Change. Guilford Press.

Jenkins, A. (2018, January 5). Half of Americans Feel Lonely, Study Finds. Fortune. https://fortune.com/2018/05/01/americans-lonely-cignastudy/

Lea, T., Amada, N., \& Jungaberle, H. (2019). Psychedelic Microdosing: A Subreddit Analysis. Journal of Psychoactive Drugs, $0(0)$, 1-12. https://doi.org/10.1080/02791072.2019.1683260

Lukianoff, G., \& Haidt, J. (2015, September). The Coddling of the American Mind. The Atlantic. https://www.theatlantic.com/magazine/ archive/2015/09/the-coddling-of-the-american-mind/399356/

Lukianoff, G., \& Haidt, J. (2018). The coddling of the American mind: How good intentions and bad ideas are setting up a generation for failure.

MacLean, K. A., Johnson, M. W., \& Griffiths, R. R. (2011)

Mystical experiences occasioned by the hallucinogen psilocybin

lead to increases in the personality domain of openness. Journal of

Psychopharmacology (Oxford, England), 25(11), 1453-1461. https://doi. org/10.1177/0269881111420188

Millière, R., Carhart-Harris, R. L., Roseman, L., Trautwein, F.-M., \& Berkovich-Ohana, A. (2018). Psychedelics, Meditation, and SelfConsciousness. Frontiers in Psychology, 9. https://doi.org/10.3389/ fpsyg.2018.01475

Nichols, D. E. (2016). Psychedelics. Pharmacological Reviews, 68(2), 264-355. https://doi.org/10.1124/pr.115.011478

Polito, V., \& Stevenson, R. J. (2019). A systematic study of microdosing psychedelics. PLOS ONE, 14(2), e0211023. https://doi org/10.1371/journal.pone.0211023

Prochazkova, L., Lippelt, D. P., Colzato, L. S., Kuchar, M., Sjoerds, Z., \& Hommel, B. (2018a). Exploring the effect of microdosing psychedelics on creativity in an open-label natural setting. Psychopharmacology. https:// doi.org/10.1007/s00213-018-5049-7

Prochazkova, L., Lippelt, D. P., Colzato, L. S., Kuchar, M., Sjoerds, Z., \& Hommel, B. (2018b). Exploring the effect of microdosing psychedelics on creativity in an open-label natural setting. Psychopharmacology, 235(12), 3401-3413. https://doi.org/10.1007/s00213-018-5049-7

Reker, G. T., \& Wong, P. T. P. (1988). Aging as an individual process: Toward a theory of personal meaning. In J. E. Birren \& V. L. Bengtson (Eds.), Emergent theories of aging (pp. 214-246). New York, NY, US: Springer Publishing Co.

Rosenbaum, D., Weissman, C., Anderson, T., Petranker, R., Dinh-Williams, L.-A., Hui, K., \& Hapke, E. (2020). Microdosing psychedelics: Demographics, practices, and psychiatric comorbidities. Journal of Psychopharmacology, 0269881120908004. https://doi. org/10.1177/0269881120908004

Ruiz, F. J. (2012). Acceptance and Commitment Therapy versus Traditional Cognitive Behavioral Therapy: A Systematic Review and Meta-analysis of Current Empirical Evidence. International Journal of Psychology, 25.

Sliwa, J. (2017, August 5). So Lonely I Could Die. Https://Www.Apa. Org. https://www.apa.org/news/press/releases/2017/08/lonely-die

Steger, M. F., Kashdan, T. B., Sullivan, B. A., \& Lorentz, D. (2008). Understanding the search for meaning in life: Personality, cognitive style and the dynamic between seeking and experiencing meaning. Journal of personality, 76(2), 199-228.

Turner, S., Mota, N., Bolton, J., \& Sareen, J. (2018). Self-medication with alcohol or drugs for mood and anxiety disorders: A narrative review of the epidemiological literature. Depression and Anxiety, 35(9), 851-860. https://doi.org/10.1002/da.22771

Twenge, J. M., Sherman, R. A., Exline, J. J., \& Grubbs, J. B. (2016) Declines in American adults' religious participation and beliefs, 1972-2014. Sage Open, 6(1), 2158244016638133

Twenge, J. M., Cooper, A. B., Joiner, T. E., Duffy, M. E., \& Binau, S.
G. (2019). Age, period, and cohort trends in mood disorder indicators and suicide-related outcomes in a nationally representative dataset, 2005-2017. Journal of abnormal psychology.

Vervaeke, J., \& Miscevic, C. M. and F. (2017). Zombies in Western Culture: A Twenty-First Century Crisis. Open Book Publishers. https://doi org/10.11647/OBP.0113

Watts, R., \& Luoma, J. B. (2020). The use of the psychological flexibility model to support psychedelic assisted therapy. Journal of Contextual Behavioral Science, 15, 92-102. https://doi.org/10.1016/j. jcbs.2019.12.004

Webb, M., Copes, H., \& Hendricks, P. S. (2019). Narrative identity, rationality, and microdosing classic psychedelics. International Journal of Drug Policy, 70, 33-39.

World Health Organisation data, prevalence of depression and anxiety disorders increased by $18.6 \%$ and $14.9 \%$ globally between 2005 and 2015 (WHO, 2017)

Yaden, D. B., Le Nguyen, K. D., Kern, M. L., Belser, A. B., Eichstaedt, J. C., Iwry, J., . . Newberg, A. B. (2017). Of roots and fruits: A comparison of psychedelic and nonpsychedelic mystical experiences. Journal of Humanistic Psychology, 57(4), 338-353. 